\title{
TRAUMATIC SHOCK. XI. INTESTINAL ABSORPTION IN HEMORRHAGIC SHOCK ${ }^{1}$
}

\author{
By MARTHA GOLDBERG AND JACOB FINE \\ (From the Surgical Research Laboratory, Beth Israel Hospital, and the Department of Surgery, \\ Harvard Medical School, Boston)
}

(Received for publication September 15, 1944)

The clinical picture of traumatic shock includes obvious disturbance of the gastro-intestinal tract. Nausea and occasional vomiting occur, particularly if fluid or food is taken. Since alimentation and peroral drug therapy apply to the patient in shock as in other illnesses, it is desirable to have some quantitative measure of the degree to which gastro-intestinal absorptive capacity is altered. Experiments in which the absorptive capacity of the small intestine in hemorrhagic shock was measured are herewith reported. The data comprise measurements of the disappearance rates of water, physiological saline solution, and 5 per cent glucose solution from loops of jejunum. They demonstrate a markedly depressed absorptive capacity of the gut during hemorrhagic shock and only partial, if any, improvement of function in the immediate recovery phase following a therapeutic transfusion.

\section{METHOD}

Normal dogs under morphine ( $2 \mathrm{mgm}$. per $\mathrm{kgm}$.) were anesthetized with ether or operated under novocaine locally. Through an abdominal incision a segment of jejunum was divided into 3 loops, each $15 \mathrm{~cm}$. in length. Catheters were tied into both open ends of each loop, one catheter for the introduction of fluid, the other for collection of fluid. With the collecting catheter filled with fluid and clamped, the capacity of each loop was determined without increasing intraluminal tension. The loops were then washed thoroughly with physiological saline, after which the abdomen was closed around the exteriorized ends of the 3 pairs of catheters. The loops were filled at zero time with water, physiological saline, and 5 per cent glucose, respectively. The capacity of the loops, filled without increase in tension, was unchanged. After 30 minutes, each loop was drained. The loop containing water was not disturbed further, but the loops containing saline solution or glucose were thoroughly washed with distilled water and the washings added to

\footnotetext{
1 The work described in this paper was done under a contract, recommended by the Committee on Medical Research, between the Office of Scientific Research and Development and Harvard University.
}

the drained fluid and the total volume noted. In the latter 2 instances, aliquots were measured for sodium chloride (1) or glucose (2), respectively.

In all dogs, blood pressure, was determined continuously by a mercury manometer and a cannula containing heparin in the left femoral artery. Venous oxygen was determined on mixed venous blood drawn from the right heart via a cannula inserted through the jugular vein.

The normal findings obtained, the dogs were then bled rapidly to a systolic blood pressure level of $50 \mathrm{~mm}$. $\mathrm{Hg}$ and maintained at approximately this level for several hours, after which (in 17 of 21 experiments), most or all of the shed heparinized blood was returned by vein to the circulation.

During the hypotensive period and after the transfusion, absorption capacity determinations were repeated for each loop.

Control observations were made on dogs prepared as described, except that only local anesthesia was used and hemorrhagic shock was not produced. Absorption studies were made for a period of 3 to 4 hours, the average duration of the shock experiments.

\section{RESULTS}

Control observations. The absorptive capacity of the loops for all 3 solutions in unshocked dogs during a period of some 4 hours after preparing the loops may vary considerably from one test to the next, but the range of variation is such that a moderate but not a sharp decline in absorptive capacity occurs as a function of time (Table I). As will be seen below, such variations are considerably less than those observed in dogs in shock.

Absorption of glucose. In morphinized laboratory dogs, glucose absorption from a jejunal loop varied widely. In general, the percentage of absorption in these animals, before shock was induced, was at a low level to begin with. This is possibly due to the fact that most of these dogs had been etherized for preparation of the loops, while the control preparations were made under local anesthesia. Nevertheless, a glance at the shift in absorptive capacity once shock is induced makes the conclusion obvious that absorptive ca- 
TABLE I

Absorptive capacity of jejunal loops in normal dogs

\begin{tabular}{c|c|c|c}
\hline Postoperative time * & Dog No. 30 & Dog No. 31 & Dog No. 33 \\
\cline { 1 - 3 } $\begin{array}{c}\text { hours } \\
\text { 5 per cent glucose }\end{array}$ in distilled water & \multicolumn{3}{|c}{ per cent } \\
1.0 & 60 & & \\
1.75 to 2.25 & 42 & 45 & 56 \\
3.0 to 3.50 & 37 & 45 & 49 \\
4.0 to 4.33 & 40 & & 48 \\
& & & \\
0.85 per cent & & & \\
saline solution & 17.5 & & 33 \\
1.0 & 0 & 19 & 27 \\
1.75 to 2.50 & 14 & 13 & 26 \\
3.0 to 3.50 & 14 & & 27 \\
4.0 to 4.33 & & & \\
Water & 33 & & 60 \\
1.0 & 20 & 67 & 56 \\
1.75 to 2.50 & 20 & 54 & 43 \\
3.0 to 3.50 & 40 & & 60 \\
4.0 to 4.33 & & & \\
\hline
\end{tabular}

* Time after preparing loops. Each determination was made upon a freshly washed loop refilled with the same volume of test material.

pacity is markedly impaired (Table II). Of 19 dogs in whom several glucose absorption determinations were obtained, 18 showed a substantial and progressive decrease in glucose absorption as shock deepened and as the blood pressure continued to fall. The nineteenth dog, which was among 4 dogs whose blood pressure level was above $60 \mathrm{~mm}$. $\mathrm{Hg}$ for a substantial period prior to transfusion, did not show a decrease. Determinations of glucose absorption after transfusion were obtained in 15 dogs of the series. Of these, 4 showed some improvement in absorptive capacity and a fifth, which also received bovine albumin, showed marked improvement. Although the blood pressure response was satisfactory in all of these, improvement did not occur in others in which this response was equally satisfactory. The absorptive capacity after transfusion generally declined from the initial post-transfusion value, whether the blood pressure remained up or declined.

Absorption of water. Water absorption from a jejunal loop likewise varies considerably among unshocked dogs recovering from ether anesthesia given for the preparation of loops (Table III). The disparity among such dogs is not so great for the absorption of water as of glucose. Of 13 dogs in which serial water absorption determinations were made during hemorrhagic shock, all showed a marked decrease in absorption as shock deepened. Four showed improvement in water absorption following blood transfusion and a fifth showed marked improvement following an infusion of 5 per cent bovine albumin. The posttransfusion capacity for water absorption was sustained whether the blood pressure did or did not decline subsequently. This is in contrast to the continuing decline in the absorptive capacity for glucose after transfusion.

Absorption of physiologic saline solution. One will note the marked difference in the absorptive capacity for physiologic saline solution between unshocked dogs emerging from ether anesthesia and unshocked dogs who had local anesthesia only (Table IV). While there are occasional instances of decline in the absorptive capacity during shock, there is in general no clearly defined effect on this function for physiologic saline. Indeed, contrary to the figures for water and glucose, not a few instances of improved absorption are noted after shock has deepened, only to decline again considerably as shock continues to deepen after an ineffective transfusion.

\section{DISCUSSION}

The volume flow of blood through the capillary circulation of the intestine in shock is less than normal (3) and the rate of flow is also below normal, if flow through the mesentery or the omentum may be taken as a gauge (4). This circumstance presumably explains the decline in the rate of absorption of intraluminal fluids and electrolytes. The absorption of glucose, however, is not a simple diffusion process across a semipermeable membrane (5). Phosphorylation must precede its absorption. That phosphorylation is disturbed in shock because of anoxia has been shown (6 to 8) in various organ systems. Since deficient blood flow and anoxia coexist in all types of shock, and since all organs are involved, including the pancreas and liver which may be unable to deliver their normal complement of digestive juices and bile, it is not improbable that the absorption of almost any normally absorbable food and fluid elements is likely to be deficient.

The absorptive capacity of the small intestine in severely bled dogs has been measured (9) and similar findings noted except for glucose, the ab- 
INTESTINAL ABSORPTION IN HEMORRHAGIC SHOCK

TABLE II

Rate of intestinal absorption of glucose

\begin{tabular}{|c|c|c|c|c|c|c|c|c|}
\hline \multicolumn{3}{|c|}{ NORMAL } & \multicolumn{3}{|c|}{ SHOCK } & \multicolumn{3}{|c|}{ AFTER THERAPY } \\
\hline Dog No. & B.P. & Absorption & Duration & B.P. & Absorption & Interval & B.P. & Absorption \\
\hline $1^{*}$ & $\begin{array}{c}\text { mm. } H_{g} \\
120\end{array}$ & $\begin{array}{c}\text { per cent } \\
38.3\end{array}$ & $\begin{array}{l}\text { hours } \\
2.0\end{array}$ & $\begin{array}{c}\mathrm{mm} \cdot \boldsymbol{H}_{\boldsymbol{g}} \\
30\end{array}$ & $\begin{array}{c}\text { per cent } \\
14.4\end{array}$ & hours & $m m \cdot H_{g}$ & per cent \\
\hline $2^{*}$ & 110 & 20.6 & 4.0 & 50 & 6.5 & & & \\
\hline $3 *$ & 110 & 32.2 & 3.0 & 65 & 25.2 & & • & \\
\hline $4^{*}$ & 120 & 56.0 & 3.0 & 55 & 45.0 & 2.25 & 80 & 26.6 \\
\hline $5^{*}$ & 125 & 76.0 & $\begin{array}{l}2.0 \\
3.5\end{array}$ & $\begin{array}{l}55 \\
40\end{array}$ & $\begin{array}{l}60.0 \\
44.0\end{array}$ & 1.50 & 100 & 42.0 \\
\hline $7 *$ & 90 & 87.5 & $\begin{array}{l}1.5 \\
3.5\end{array}$ & $\begin{array}{l}70 \\
55\end{array}$ & $\begin{array}{l}37.5 \\
27.7\end{array}$ & 1.50 & 90 & 19.9 \\
\hline $8^{*}$ & 95 & 84.7 & 2.0 & 30 & 24.5 & 2.5 & 115 & 39.7 \\
\hline $9^{*}$ & 100 & 53.3 & 2.5 & 30 & 6.5 & & & \\
\hline $10^{*}$ & 85 & 19.9 & $\begin{array}{l}2.0 \\
3.75\end{array}$ & $\begin{array}{l}50 \\
65\end{array}$ & $\begin{array}{r}19.5 \\
4.5\end{array}$ & $\begin{array}{c}0.5 \\
1.75 \\
\text { alb. i.v. } \\
1.0\end{array}$ & $\begin{array}{l}85 \\
75 \\
90\end{array}$ & $\begin{array}{c}8.7 \\
8.7 \\
26.6\end{array}$ \\
\hline $11^{*}$ & 100 & 19.2 & $\begin{array}{l}2.25 \\
3.25 \\
4.0 \\
\end{array}$ & $\begin{array}{l}45 \\
50 \\
30\end{array}$ & $\begin{array}{l}5.2 \\
4.6 \\
0\end{array}$ & $\begin{array}{l}0.5 \\
1.5\end{array}$ & $\begin{array}{l}90 \\
70\end{array}$ & $\begin{array}{l}5.2 \\
1.2\end{array}$ \\
\hline $12^{*}$ & 80 & 20.6 & $\begin{array}{l}1.0 \\
1.75 \\
2.5\end{array}$ & $\begin{array}{l}65 \\
60 \\
45\end{array}$ & $\begin{array}{l}22.8 \\
12.5 \\
20.6\end{array}$ & $\begin{array}{l}0.75 \\
1.5\end{array}$ & $\begin{array}{l}45 \\
30\end{array}$ & $\begin{array}{l}15.3 \\
10.5\end{array}$ \\
\hline $13^{*}$ & $\begin{array}{r}100 \\
95\end{array}$ & $\begin{array}{l}47.8 \\
42.6\end{array}$ & $\begin{array}{l}1.0 \\
2.0 \\
2.5\end{array}$ & $\begin{array}{l}60 \\
75 \\
40\end{array}$ & $\begin{array}{l}43.0 \\
31.8 \\
14.8\end{array}$ & $\begin{array}{c}1.5 \\
2.25 \\
3.25 \\
\text { 2nd trans. } \\
1.0 \\
2.0\end{array}$ & $\begin{array}{l}85 \\
90 \\
70 \\
90 \\
90\end{array}$ & $\begin{array}{r}18.7 \\
12.4 \\
22.6 \\
8.1 \\
15.0\end{array}$ \\
\hline $14^{*}$ & $\begin{array}{l}110 \\
115\end{array}$ & $\begin{array}{l}20.6 \\
15.5\end{array}$ & $\begin{array}{l}1.0 \\
2.25 \\
3.0\end{array}$ & $\begin{array}{l}75 \\
65 \\
60\end{array}$ & $\begin{array}{l}23.8 \\
44.3 \\
48.6\end{array}$ & $\begin{array}{l}1.5 \\
2.0\end{array}$ & $\begin{array}{l}130 \\
110\end{array}$ & $\begin{array}{l}22.0 \\
19.6\end{array}$ \\
\hline $16^{*}$ & 90 & 14.9 & $\begin{array}{l}0.75 \\
2.5\end{array}$ & $\begin{array}{l}50 \\
35\end{array}$ & $\begin{array}{r}13.1 \\
6.6\end{array}$ & 2.0 & 40 & $6.6 t$ \\
\hline $17^{*}$ & $\begin{array}{l}120 \\
110\end{array}$ & $\begin{array}{l}6.5 \\
6.5\end{array}$ & 1.75 & 45 & 0 & $\begin{array}{l}0.75 \\
2.5\end{array}$ & $\begin{array}{r}110 \\
30\end{array}$ & $\begin{array}{l}0 \\
6.5\end{array}$ \\
\hline $19^{*}$ & $\begin{array}{l}105 \\
105\end{array}$ & $\begin{array}{l}33.2 \\
28.0\end{array}$ & 1.5 & 70 & 16.3 & $\begin{array}{l}1.75 \\
3.75\end{array}$ & $\begin{array}{r}105 \\
85\end{array}$ & $\begin{array}{l}27.1 \\
21.9\end{array}$ \\
\hline $20 t$ & $\begin{array}{l}130 \\
120\end{array}$ & $\begin{array}{l}30.8 \\
26.7\end{array}$ & 1.5 & 20 & 14.0 & $\begin{array}{l}1.0 \\
2.5\end{array}$ & $\begin{array}{l}95 \\
50\end{array}$ & $\begin{array}{l}20.0 \\
11.0\end{array}$ \\
\hline $21 \dagger$ & $\begin{array}{l}130 \\
140\end{array}$ & $\begin{array}{l}41.5 \\
39.0\end{array}$ & $\begin{array}{l}1.0 \\
1.75\end{array}$ & $\begin{array}{l}70 \\
50\end{array}$ & $\begin{array}{l}26.5 \\
12.1\end{array}$ & $\begin{array}{l}1.25 \\
2.25\end{array}$ & $\begin{array}{l}120 \\
120\end{array}$ & $\begin{array}{r}28.3 \\
6.5\end{array}$ \\
\hline $32 \dagger$ & $\begin{array}{l}100 \\
100\end{array}$ & $\begin{array}{l}60.9 \\
57.1\end{array}$ & $\begin{array}{l}0.75 \\
1.5\end{array}$ & $\begin{array}{l}45 \\
40\end{array}$ & $\begin{array}{l}36.4 \\
14.0\end{array}$ & $\begin{array}{l}0.75 \\
1.5\end{array}$ & $\begin{array}{l}100 \\
100\end{array}$ & $\begin{array}{l}41.2 \\
35.0\end{array}$ \\
\hline
\end{tabular}

* Ether anesthesia.

$\dagger$ Local anesthesia.

‡ Saline and pitressin i.v. after blood. 
TABLE III

Rate of intestinal absorption of water

\begin{tabular}{|c|c|c|c|c|c|c|c|c|}
\hline \multicolumn{3}{|c|}{ NORMAL } & \multicolumn{3}{|c|}{ sHocx } & \multicolumn{3}{|c|}{ AFTER THERAPY } \\
\hline Dog No. & B.P. & Absorption & Duration & B.P. & Abeorption & Interval & B.P. & Abeorption \\
\hline $8^{*}$ & $\operatorname{mm.~}_{95} H_{8}$ & $\begin{array}{c}\text { per cent } \\
77.7\end{array}$ & $\begin{array}{l}\text { hours } \\
2.0\end{array}$ & $\begin{array}{c}\mathrm{mm}_{30} \mathrm{Hg}_{\mathrm{g}} \\
\end{array}$ & $\begin{array}{c}\text { per cent } \\
28.6\end{array}$ & $\begin{array}{l}\text { hours } \\
2.5\end{array}$ & ${ }_{115}^{m m .} B_{g}$ & $\begin{array}{c}\text { per cont } \\
50.0\end{array}$ \\
\hline $9^{*}$ & 100 & 83.4 & 2.5 & 30 & 33.3 & & & \\
\hline $10^{*}$ & 85 & 50.0 & $\begin{array}{l}2.0 \\
3.75\end{array}$ & $\begin{array}{l}50 \\
65\end{array}$ & $\begin{array}{l}17.9 \\
14.3\end{array}$ & $\begin{array}{c}0.5 \\
1.75 \\
\text { alb. i.v. } \\
1.0\end{array}$ & $\begin{array}{l}85 \\
75 \\
90\end{array}$ & $\begin{array}{l}14.3 \\
21.4 \\
\\
53.5\end{array}$ \\
\hline $11^{*}$ & 100 & 87.0 & $\begin{array}{l}2.25 \\
3.25 \\
4.0\end{array}$ & $\begin{array}{l}45 \\
50 \\
30\end{array}$ & $\begin{array}{l}26.8 \\
36.8 \\
20.0\end{array}$ & $\begin{array}{l}0.5 \\
1.5\end{array}$ & $\begin{array}{l}90 \\
70\end{array}$ & $\begin{array}{l}\mathbf{0} \\
0\end{array}$ \\
\hline $12^{*}$ & 80 & 63.5 & $\begin{array}{l}1.0 \\
1.75 \\
2.50\end{array}$ & $\begin{array}{l}65 \\
60 \\
45\end{array}$ & $\begin{array}{l}63.7 \\
46.7 \\
33.3\end{array}$ & $\begin{array}{l}0.75 \\
1.50\end{array}$ & $\begin{array}{l}45 \\
30\end{array}$ & $\begin{array}{l}66.7 \\
53.5\end{array}$ \\
\hline $13^{*}$ & $\begin{array}{r}100 \\
95\end{array}$ & $\begin{array}{l}100 \\
100\end{array}$ & $\begin{array}{l}1.0 \\
2.0 \\
2.5\end{array}$ & $\begin{array}{l}60 \\
75 \\
40\end{array}$ & $\begin{array}{l}85.8 \\
50.0 \\
14.2\end{array}$ & $\begin{array}{c}1.50 \\
2.25 \\
3.25 \\
\text { 2nd trans. } \\
1.0 \\
2.0\end{array}$ & $\begin{array}{l}85 \\
90 \\
70 \\
90 \\
90\end{array}$ & $\begin{array}{l}28.5 \\
64.2 \\
71.4 \\
\\
56.2 \\
56.2\end{array}$ \\
\hline $14^{*}$ & $\begin{array}{l}110 \\
115\end{array}$ & $\begin{array}{l}86.5 \\
76.5\end{array}$ & $\begin{array}{l}1.0 \\
2.25 \\
3.0\end{array}$ & $\begin{array}{l}75 \\
65 \\
60\end{array}$ & $\begin{array}{r}60.0 \\
7.9 \\
7.9\end{array}$ & $\begin{array}{l}1.5 \\
2.0\end{array}$ & $\begin{array}{l}130 \\
110\end{array}$ & $\begin{array}{r}8.6 \\
10.0\end{array}$ \\
\hline $16^{*}$ & 90 & 100 & $\begin{array}{l}0.25 \\
2.50\end{array}$ & $\begin{array}{l}50 \\
35\end{array}$ & $\begin{array}{r}33.2 \\
6.7\end{array}$ & 2.0 & 40 & $0 \ddagger$ \\
\hline $17^{*}$ & $\begin{array}{l}120 \\
110\end{array}$ & $\begin{array}{l}90.0 \\
66.6\end{array}$ & $\begin{array}{l}0.75 \\
1.75\end{array}$ & $\begin{array}{l}70 \\
45\end{array}$ & $\begin{array}{l}43.3 \\
33.3\end{array}$ & $\begin{array}{l}0.75 \\
2.50\end{array}$ & $\begin{array}{r}110 \\
30\end{array}$ & $\begin{array}{l}23.3 \\
26.5\end{array}$ \\
\hline $18^{*}$ & $\begin{array}{l}100 \\
100\end{array}$ & $\begin{array}{l}75.0 \\
55.0\end{array}$ & $\begin{array}{l}1.0 \\
2.0\end{array}$ & $\begin{array}{l}60 \\
40\end{array}$ & $\begin{array}{l}50.0 \\
25.0\end{array}$ & 1.0 & 110 & 25.0 \\
\hline $19 *$ & $\begin{array}{l}105 \\
105\end{array}$ & $\begin{array}{l}66.0 \\
33.0\end{array}$ & 1.5 & 70 & 0 & $\begin{array}{l}1.75 \\
3.75\end{array}$ & $\begin{array}{l}95 \\
50\end{array}$ & $\begin{array}{l}6.7 \\
6.7\end{array}$ \\
\hline $20 \dagger$ & $\begin{array}{l}130 \\
120\end{array}$ & $\begin{array}{l}80.0 \\
55.0\end{array}$ & 1.5 & 20 & 30.0 & $\begin{array}{l}1.0 \\
2.5\end{array}$ & $\begin{array}{l}95 \\
50\end{array}$ & $\begin{array}{l}0 \\
5.0\end{array}$ \\
\hline $21 \dagger$ & $\begin{array}{l}130 \\
140\end{array}$ & $\begin{array}{l}80.0 \\
50.0\end{array}$ & $\begin{array}{l}1.0 \\
1.75\end{array}$ & $\begin{array}{l}70 \\
50\end{array}$ & $\begin{array}{l}10.0 \\
10.0\end{array}$ & $\begin{array}{l}1.25 \\
2.25\end{array}$ & $\begin{array}{l}120 \\
120\end{array}$ & $\begin{array}{c}10.0 \\
0\end{array}$ \\
\hline $32 \dagger$ & $\begin{array}{l}100 \\
100\end{array}$ & $\begin{array}{l}92.6 \\
92.6\end{array}$ & $\begin{array}{l}0.75 \\
1.50\end{array}$ & $\begin{array}{l}45 \\
40\end{array}$ & $\begin{array}{l}57.4 \\
20.0\end{array}$ & $\begin{array}{l}0.75 \\
1.50\end{array}$ & $\begin{array}{l}100 \\
100\end{array}$ & $\begin{array}{l}40 \\
40\end{array}$ \\
\hline
\end{tabular}

* Ether anesthesia.

† Local anesthesia.

‡ Saline and pitressin i.v. after blood.

sorption of which was found uninfluenced by the hemorrhage. These experiments are not strictly comparable to ours, since no effort was made to produce a sustained state of shock; in addition, only one posthemorrhage determination was made while we made successive absorption tests as shock continued. The relative ease of absorption of physiologic saline after hemorrhage was first noted by these workers and we are able to confirm this finding. The explanation for this finding may lie in the greater need for physiologic saline in shock than for water or isotonic glucose and in the small amount of work and therefore lower peripheral circulatory efficiency required for its absorption than for the absorption of water or glucose solution. 
TABLE IV

Rate of intestinal absorption of saline

\begin{tabular}{|c|c|c|c|c|c|c|c|c|}
\hline \multicolumn{3}{|c|}{ NORMAL. } & \multicolumn{3}{|c|}{ sHocx } & \multicolumn{3}{|c|}{ AFTER THERAPY } \\
\hline Dor No. & B.P. & Abeorption & Duration & B.P. & Absorption & Interval & B.P. & Absorption \\
\hline $8^{*}$ & $\begin{array}{c}\text { mm. } \\
95\end{array}$ & $\begin{array}{c}\text { per cent } \\
70.0\end{array}$ & $\begin{array}{l}\text { hours } \\
2.0\end{array}$ & $\begin{array}{c}m m . B g \\
30\end{array}$ & $\begin{array}{c}\text { per cent } \\
32.1\end{array}$ & $\begin{array}{l}\text { hours } \\
2.5\end{array}$ & $\begin{array}{c}m m . H_{g} \\
115\end{array}$ & $\begin{array}{c}\text { per cent } \\
39.7\end{array}$ \\
\hline $10^{*}$ & 85 & 14.7 & 2.0 & 50 & 20.3 & $\begin{array}{c}0.5 \\
1.75 \\
\text { alb. i.v. } \\
1.0\end{array}$ & $\begin{array}{l}85 \\
75 \\
90\end{array}$ & $\begin{array}{r}9.1 \\
7.6 \\
11.9\end{array}$ \\
\hline $11^{*}$ & 100 & 0 & $\begin{array}{l}2.25 \\
3.25 \\
4.0\end{array}$ & $\begin{array}{l}45 \\
50 \\
30\end{array}$ & $\begin{array}{l}22.8 \\
17.1 \\
10.6\end{array}$ & $\begin{array}{l}0.5 \\
1.5\end{array}$ & $\begin{array}{l}90 \\
70\end{array}$ & $\begin{array}{r}20.3 \\
8.9\end{array}$ \\
\hline $12^{*}$ & 80 & 6.5 & $\begin{array}{l}1.0 \\
1.75 \\
2.5\end{array}$ & $\begin{array}{l}65 \\
60 \\
45\end{array}$ & $\begin{array}{l}18.7 \\
25.9 \\
27.6\end{array}$ & $\begin{array}{l}0.75 \\
1.5\end{array}$ & $\begin{array}{l}45 \\
30\end{array}$ & $\begin{array}{r}2.4 \\
10.6\end{array}$ \\
\hline $13^{*}$ & $\begin{array}{r}100 \\
95\end{array}$ & $\begin{array}{r}15.9 \\
8.8\end{array}$ & $\begin{array}{l}1.0 \\
2.0 \\
2.5\end{array}$ & $\begin{array}{l}60 \\
75 \\
40\end{array}$ & $\begin{array}{c}14.2 \\
0 \\
0\end{array}$ & $\begin{array}{c}1.5 \\
2.25 \\
3.25 \\
\text { 2nd trans. } \\
1.0 \\
2.0\end{array}$ & $\begin{array}{l}85 \\
90 \\
70 \\
90 \\
90\end{array}$ & $\begin{array}{c}0 \\
0 \\
0 \\
10.7 \\
7.9\end{array}$ \\
\hline $14^{*}$ & $\begin{array}{l}110 \\
115\end{array}$ & $\stackrel{0}{11.7}$ & $\begin{array}{l}1.0 \\
2.25 \\
3.0\end{array}$ & $\begin{array}{l}75 \\
65 \\
60\end{array}$ & $\begin{array}{r}20.1 \\
17.6 \\
3.4\end{array}$ & $\begin{array}{l}1.5 \\
2.0\end{array}$ & $\begin{array}{l}130 \\
110\end{array}$ & $\begin{array}{r}5.9 \\
65.6\end{array}$ \\
\hline $16^{*}$ & 90 & 45.0 & $\begin{array}{l}0.75 \\
2.50\end{array}$ & $\begin{array}{l}50 \\
35\end{array}$ & $\begin{array}{r}43.0 \\
9.2\end{array}$ & 2.0 & 40 & $0 \neq$ \\
\hline $17^{*}$ & $\begin{array}{l}120 \\
110\end{array}$ & $\begin{array}{l}0 \\
4.6\end{array}$ & $\begin{array}{l}0.75 \\
1.75\end{array}$ & $\begin{array}{l}70 \\
45\end{array}$ & $\begin{array}{l}14.2 \\
15.9\end{array}$ & $\begin{array}{l}0.75 \\
2.50\end{array}$ & $\begin{array}{r}110 \\
30\end{array}$ & $\begin{array}{r}15.9 \\
9.2\end{array}$ \\
\hline $18^{*}$ & $\begin{array}{l}100 \\
100\end{array}$ & $\begin{array}{c}17.0 \\
0\end{array}$ & $\begin{array}{l}1.0 \\
2.0\end{array}$ & $\begin{array}{l}60 \\
40\end{array}$ & $\begin{array}{l}6.8 \\
0\end{array}$ & & & \\
\hline $19^{*}$ & $\begin{array}{l}105 \\
105\end{array}$ & $\begin{array}{l}0 \\
1.9\end{array}$ & 1.5 & 70 & 13.1 & $\begin{array}{l}1.75 \\
3.75\end{array}$ & $\begin{array}{r}105 \\
85\end{array}$ & $\begin{array}{l}9.8 \\
1.9\end{array}$ \\
\hline $20 \dagger$ & $\begin{array}{l}130 \\
120\end{array}$ & $\begin{array}{l}25.0 \\
25.0\end{array}$ & 1.5 & 20 & 17.0 & $\begin{array}{l}1.0 \\
2.5\end{array}$ & $\begin{array}{l}95 \\
50\end{array}$ & $\begin{array}{l}8.0 \\
8.0\end{array}$ \\
\hline $21 \dagger$ & $\begin{array}{l}130 \\
140\end{array}$ & $\begin{array}{l}40.0 \\
47.5\end{array}$ & $\begin{array}{l}1.0 \\
1.75\end{array}$ & $\begin{array}{l}70 \\
50\end{array}$ & $\begin{array}{l}35.5 \\
25.6\end{array}$ & $\begin{array}{l}1.25 \\
2.25\end{array}$ & $\begin{array}{l}120 \\
120\end{array}$ & $\begin{array}{l}6.1 \\
0\end{array}$ \\
\hline $22 \dagger$ & $\begin{array}{l}120 \\
120\end{array}$ & $\begin{array}{l}27.9 \\
43.0\end{array}$ & $\begin{array}{l}0.75 \\
1.50\end{array}$ & $\begin{array}{l}\mathbf{5 0} \\
\mathbf{3 0}\end{array}$ & $\begin{array}{c}30.8 \\
0\end{array}$ & $\begin{array}{l}1.0 \\
2.5\end{array}$ & $\begin{array}{l}105 \\
110\end{array}$ & $\begin{array}{r}4.8 \\
33.3\end{array}$ \\
\hline $23 \dagger$ & $\begin{array}{l}120 \\
120\end{array}$ & $\begin{array}{l}50.7 \\
19.0\end{array}$ & $\begin{array}{l}0.75 \\
1.50\end{array}$ & $\begin{array}{l}70 \\
40\end{array}$ & $\begin{array}{l}23.6 \\
61.3\end{array}$ & $\begin{array}{l}1.0 \\
3.0\end{array}$ & $\begin{array}{l}140 \\
120\end{array}$ & $\begin{array}{l}34.6 \\
47.8\end{array}$ \\
\hline $24 \dagger$ & $\begin{array}{l}150 \\
150\end{array}$ & $\begin{array}{r}15.4 \\
1.7\end{array}$ & 0.75 & 65 & 18.8 & & . & \\
\hline $26 t$ & $\begin{array}{l}140 \\
140\end{array}$ & $\begin{array}{l}37.7 \\
32.4\end{array}$ & $\begin{array}{l}0.75 \\
1.50\end{array}$ & $\begin{array}{l}45 \\
35\end{array}$ & $\begin{array}{l}32.4 \\
42.0\end{array}$ & $\begin{array}{l}0.75 \\
1.50\end{array}$ & $\begin{array}{l}90 \\
70\end{array}$ & $\begin{array}{l}7.5 \\
3.7\end{array}$ \\
\hline $27 \dagger$ & $\begin{array}{l}130 \\
120\end{array}$ & $\begin{array}{l}32.4 \\
30.0\end{array}$ & $\begin{array}{l}0.50 \\
1.50 \\
2.25\end{array}$ & $\begin{array}{l}60 \\
45 \\
45\end{array}$ & $\begin{array}{l}20.3 \\
31.6 \\
26.4\end{array}$ & $\begin{array}{l}0.75 \\
2.0\end{array}$ & $\begin{array}{l}105 \\
105\end{array}$ & $\begin{array}{r}20.7 \\
7.9\end{array}$ \\
\hline 29At & $\begin{array}{l}120 \\
115\end{array}$ & $\begin{array}{l}51.0 \\
56.0\end{array}$ & $\begin{array}{l}0.75 \\
2.25\end{array}$ & $\begin{array}{l}70 \\
45\end{array}$ & $\begin{array}{l}43.5 \\
33.2\end{array}$ & $\begin{array}{l}0.50 \\
1.50\end{array}$ & $\begin{array}{l}110 \\
110\end{array}$ & $\begin{array}{r}5.9 \\
28.9\end{array}$ \\
\hline $29 \mathrm{~B} \dagger$ & $\begin{array}{l}120 \\
115\end{array}$ & $\begin{array}{l}18.8 \\
35.2\end{array}$ & $\begin{array}{l}0.75 \\
2.25\end{array}$ & $\begin{array}{l}70 \\
45\end{array}$ & $\begin{array}{l}28.8 \\
21.2\end{array}$ & $\begin{array}{l}0.5 \\
1.5\end{array}$ & $\begin{array}{l}110 \\
110\end{array}$ & $\begin{array}{l}26.8 \\
30.0\end{array}$ \\
\hline $29 \mathrm{C} \dagger$ & $\begin{array}{l}120 \\
115\end{array}$ & $\begin{array}{l}37.0 \\
37.8\end{array}$ & $\begin{array}{l}0.75 \\
2.25\end{array}$ & $\begin{array}{l}70 \\
45\end{array}$ & $\begin{array}{l}35.2 \\
17.2\end{array}$ & $\begin{array}{l}0.5 \\
1.5\end{array}$ & $\begin{array}{l}110 \\
110\end{array}$ & $\begin{array}{r}9.4 \\
14.1\end{array}$ \\
\hline
\end{tabular}

\footnotetext{
* Ether anesthesia. † Local anesthesia. $\quad$ † Saline and pitressin i.v. after blood.
} 


\section{CONCLUSIONS}

In hemorrhagic shock, the small intestine is progressively deficient in its absorptive capacity for water and isotonic glucose. After transfusion, whether effective or not, some improvement in this function may be observed, but full recovery is not observed within the time interval of these experiments.

The absorption of physiologic saline is not clearly affected by the institution of hemorrhagic shock until the advanced stage of shock is reached.

\section{BIBLIOGRAPHY}

1. Whitehorn, J. C., A system of blood analysis. Simplified method for the determination of chlorides in blood or plasma. J. Biol. Chem., 1920-21, 45, 449.
2. Benedict, S. R., A method for the estimation of reducing sugars. J. Biol. Chem., 1911, 9, 57.

3. Blalock, A., and Levy, S. E., The effect of hemorrhage, intestinal trauma and histamine on the partition of the blood stream. Am. J. Physiol., 1937, 118, 734.

4. Fine, J., Seligman, A., and Frank, H. A., Unpublished data.

5. Verzár, F., and McDougall, E. J., Absorption from the Intestine. Longmans, Green and Co., London, 1936.

6. Govier, W. M., and Grieg, M. E., Personal communication.

7. Alexander, B., The chemical determination of thiamine and cocarboxylase in biological material. J. Biol. Chem., 1943, 151, 455.

8. Furchgott, R. F., and Schorr, E., Phosphate exchange in resting cardiac muscle as indicated by radioactivity studies. J. Biol. Chem., 1943, 151, 65.

9. Van Liere, E. J., Northup, D. W., and Sleeth, C. K., The effect of acute hemorrhage on absorption from small intestine. Am. J. Physiol., 1938, 124, 102. 